\title{
Patch-clamp detection of macromolecular translocation along nuclear pores
}

J.O. Bustamante and W.A. Varanda
Departamento de Fisiologia, Faculdade de Medicina de Ribeirão Preto, Universidade de São Paulo, Ribeirão Preto, SP, Brasil

\section{Correspondence J.O. Bustamante \\ Departamento de Fisiologia FMRP, USP \\ Av. Bandeirantes, 3900 14049-900 Ribeirão Preto, SP Brasil \\ Fax: 55 (016) 633-0017 \\ E-mail: jobusta@rfi.fmrp.usp.br and wvaranda@fmrp.usp.br \\ Presented at the XII Annual Meeting of the Federação de Sociedades de Biologia Experimental, Caxambu, MG, Brasil, August 27-30, 1997. \\ J.O. Bustamante and W.A. Varanda were recipients of grants from MRC, Canada and FAPESP.}

Received August 8, 1997 Accepted November 11, 1997

\section{Abstract}

The present paper reviews the application of patch-clamp principles to the detection and measurement of macromolecular translocation along the nuclear pores. We demonstrate that the tight-seal 'gigaseal' between the pipette tip and the nuclear membrane is possible in the presence of fully operational nuclear pores. We show that the ability to form a gigaseal in nucleus-attached configurations does not mean that only the activity of channels from the outer membrane of the nuclear envelope can be detected. Instead, we show that, in the presence of fully operational nuclear pores, it is likely that the large-conductance ion channel activity recorded derives from the nuclear pores. We conclude the technical section with the suggestion that the best way to demonstrate that the nuclear pores are responsible for ion channel activity is by showing with fluorescence microscopy the nuclear translocation of ions and small molecules and the exclusion of the same from the cisterna enclosed by the two membranes of the envelope. Since transcription factors and mRNAs, two major groups of nuclear macromolecules, use nuclear pores to enter and exit the nucleus and play essential roles in the control of gene activity and expression, this review should be useful to cell and molecular biologists interested in understanding how patch-clamp can be used to quantitate the translocation of such macromolecules into and out of the nucleus.

\section{Introduction}

In the present review we attempt to explain how the patch-clamp technique can be used to detect and measure macromolecular translocation (MMT) by using both qualitative and quantitative analysis of gated ion flow along the nuclear pore complex (NPC). In doing so, we hope that the concepts used can be extrapolated to other systems such as those of channels from other organellar membranes. The mechanisms of NPC-mediated
Key words

- Nuclear pores

- Nuclear envelope

- Cell nucleus

- Nucleocytoplasmic transport

- Macromolecular transport

- Gene activity

- Gene expression

- Transcriptional control

- Ion channels

- Patch-clamp

- Measurement 
patch-clamp recording of the large-conductance ion channel activity at the nuclear envelope (NE) represents gating of the NPC channel (NPCC, the central NPC hole) and how this measurement can be used to investigate NPC-MMT. We hope that this balancing-act, tight-rope approach, although trivial at times for one or another group of scientists (i.e., electrophysiologists or cell biologists), contributes to the development of new methodologies for the study of the mechanisms of gene control by imported transcription factors and of gene expression by the export of messenger RNAs (mRNAs). Both of these processes are relevant to the maintenance of normal cell function and to the development of various cellular pathologies and programmed cell death. Space limitations restrict the number of publications cited in the present review. For this reason, we refer the reader to further reading on these specialized areas.

\section{Nuclear pore ion channel behavior}

\section{Ion channels and ion channel activity}

Here we review some basic concepts on ion channels. The more demanding reader may consult other sources (e.g., 1). The simplest channel system is one that switches in a binary scheme between two states: open and closed, with values of, say, 1 and 0 (picoamperes, picosiemens, etc.). In the steady state, this simple two-state system exhibits open and closed probabili-

\footnotetext{
Abbreviations and acronyms:

AP-1: activator protein-1, a transcription factor complex consisting of c-Fos and c-Jun

AP-1/c-Jun: the c-Jun component of AP-1

ATP and GTP: adenosine and guanosine triphosphate cAMP: cyclic adenosine monophosphate

CFTR: cystic fibrosis transmembrane conductance regulator

B-PE and FITC: B-phycoerythrin and fluorescein

isothiocyanate; two fluorescent probes

INM and ONM: inner and outer nuclear membranes of the nuclear envelope

$\mathrm{IP}_{3}$ : inositol 1,4,5-trisphosphate

mAb414: monoclonal antibody 414; raised against the nuclear pore
}

ties, $p_{o}$ and $p_{c}$, such that:

$$
p_{o}+p_{c}=1
$$

Although a popular term, ion channel activity has both qualitative and quantitative connotations. Channel activity can be taken to be the set of all those parameters which determine the observable or measured quantity which, in patch-clamp, is the time-dependent electrical current, $i(t)$, carried out by the ions moving along the channel. At each point in time, the current will be determined by the value of the conductance, $\gamma$.

$i(V, t)=\gamma(V, t) V(t)$

where the parentheses next to the 'dependent' variables indicate that the variable is a function of the 'independent' variables (e.g., $V(t)$ indicates that $V$ is a function of $t)$.

In a first, gross approximation, if we think of the channel as a hollow cylinder, then the channel conductance is related to the cylinder geometry and electrolyte resistivity, $\rho$,

$\gamma=A /(l \rho)$ or $\gamma=\pi r^{2} /(l \rho)$

where $A$ is the cross-sectional area of the cylinder and $r$ and $l$ are the radius and length of the cylinder. Equation 3 summarizes the relationship between conductance, area, length and resistivity of the channel hole. The wider and shorter the channel, the larger the conductance. But, what equation 3 does not describe is the gating mechanism, which could be represented as a multiplying term whose values are either 0 or 1 . We could use

\footnotetext{
MMT: macromolecular translocation

NE: nuclear envelope

$\mathrm{NF}-\mathrm{\kappa B}$ : the transcription factor named nuclear factor kappa B

NLS: nuclear localization signal

NPC and NPCC: nuclear pore complex and its central

channel or hole

NPC-MMT: NPC-mediated MMT

PKA: cAMP-dependent protein kinase

Ran/TC4: a small GTPase involved in NPC-MMT

RNA and mRNA: ribonucleic acid and messenger RNA

SP1: a transcription factor

TATA: a deoxynucleotide sequence used here in connection to the transcription factor that binds to it: the TATA-binding protein
} 
a time-dependent Dirac's delta function, $\delta=$ $\delta(t)$, widely used to represent 0 everywhere except at a single point (which we envision as that representing the proper gating conditions):

$\gamma=\delta(t)[A /(l \rho)]$ or $\gamma=\delta(t)\left[\pi r^{2} /(l \rho)\right]$

In a random, stochastic system such as an ion channel switching between the open and closed states, the macroscopic behavior of the system is represented by time average quantities which give a measure of how the macroscopic system (the collection of single, microscopic units) works. For example, the time average channel conductance, $\langle\gamma\rangle$ would be given by:

$\langle\gamma\rangle=\langle\delta(t)\rangle[A /(l \rho)]$ or

$\langle\gamma\rangle=\langle\delta(t)\rangle\left[\pi r^{2} /(l \rho)\right]$

which can also be written as:

$\langle\gamma\rangle=p_{o}[A /(l \rho)]$ or $\left\langle\gamma=p_{o}\left[\pi r^{2} /(l \rho)\right]\right.$

Thus, the average ion current can be written as:

$\langle i(V)\rangle=\langle\gamma\rangle V$

where $V=V(t)$ is the applied voltage, which in patch-clamp is most of the time conveniently set to be constant. If $p_{o}$, and $V$, as well as channel geometry and resistivity of environment (i.e., $\rho$ ), do not change with time or voltage, then:

$\left\langle i(t)>=p_{o} \gamma V\right.$

Together, these microscopic and macroscopic parameters give a measure of channel activity, function and behavior. The more frequently the channel is open (i.e., the higher the $p_{o}$ ), and/or the greater the conductance and voltage, $\gamma$ and $V$, the greater the value of the average current, $\langle i\rangle$. Here lies the power of patch-clamp. While patch-clamp allows precise determination of the parameters that determine $\langle i\rangle$ changes, macroscopic measurements (such as whole-nucleus voltageclamp which detects whole nucleus ion current or fluorescence microscopy which detects integrated changes in probe concentration) do not permit such determination because a larger value of $\langle i\rangle$ can be obtained with either greater $p_{o}, \gamma$, or $V$, individually.

Figure 1 illustrates an equivalent circuit for an organellar ion channel with negligible reversal potential ( $V_{\text {rev }}$, the value of voltage at which the ion current reverses direction). The channel components are delimited by the rectangular box formed by the discontinued line. The channel is represented by a resistor or conductor (i.e., the vertical, solid line rectangular box represents the channel cylinder) plus a gating mechanism (a switch). The organelle electrical potential, $V_{\text {organelle }}$, is represented by the pair of thin-and-thick horizontal lines near the bottom. The voltage in the system is measured with reference to the bath potential, $V_{\text {reference }}$ or $V_{\text {bath }}$ (represented as the thick horizontal line at the bottom). The pipette potential, $V_{\text {pipette, }}$, is applied at one side of the channel. The measured quantity, the pipette current, $i_{\text {pipette}}$, is given by:

$i_{\text {pipette }}=\gamma\left(V_{\text {pipette }}-V_{\text {organelle }}\right)$

\footnotetext{
Variables:

$i$ and $\gamma$ : current and conductance of a single ion channel; microscopic variables

$I$ and $\Gamma$ : current and conductance of a channel population; macroscopic variables

$N$ : number of functional units in the ion channel population $p_{o}$ and $p_{c}$ : open and close probabilities of an ion channel

$P_{o}$ and $P_{c}$ : open and close probabilities of the ion channel population

$\tau$ : time constant of decay

$R, V$ and $t$ : electrical resistance, voltage and time

$V_{\text {rev }}$ : reversal potential, voltage at which the ion current

changes direction
}

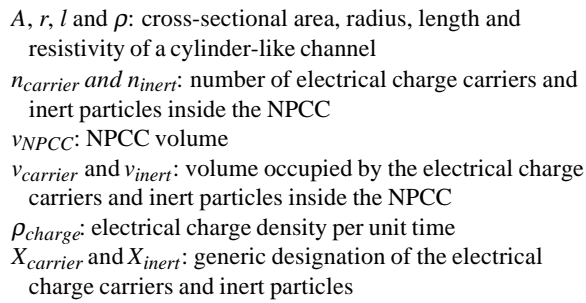




\section{Essential features of the NPC}

The NPC is the only direct pathway connecting nucleus and cytoplasm (e.g., 2-6). Under electron microscopy, the NPC is one of the most conspicuous features of the NE (e.g., 7,8). The NPC stands out as the most prominent structure in topological imaging of the NE with scanning electron microscopy (e.g., 9) and atomic force microscopy (e.g., 10). This supramolecular structure consists of many proteins which together have a mass estimated at about $124 \mathrm{MDa}$ (megadaltons, e.g., 8). Figure 2 shows a 3-D reconstruction model generated with electron microscopy data (from Ref. 8). The figure displays the putative plug in the center in a fuzzy, transparent fashion to indicate that the concept of the plug is not clear (8). Cytoplasmic filaments (vertical rod-like filaments) as well as nucleoplasmic ones (convergent at the bottom) are shown. The NPC spans the two membranes of the NE: the outer and the inner nuclear membranes (ONM and INM, respectively). This feature is es-

Figure 1 - Equivalent electrical circuit for an organelle ion channel. The model is drawn for an organelle with a simple membrane ( vis à vis a dual membrane like the nuclear envelope). The channel itself is delimited by the discontinued line. The channel is represented by a resistor or conductor (i.e., the rectangular box represents the channel cylinder) plus a gating mechanism (a switch). Allowance has been made for organelle electrical potential, $V_{\text {organelle }}$ (represented by the pair of thin-and-thick horizontal lines near the bottom). The voltage in the system is measured with reference to the bath potential, $V_{\text {reference }}$ (represented as the thick horizontal line at the bottom). The pipette potential, $V_{\text {pipette }}$, is applied to one side of the channel and the resulting current, i $i_{\text {pipette }}$ measured. The reversal potential, $V_{\text {rev }}$, for the ion current has been assumed negligible (see 31).

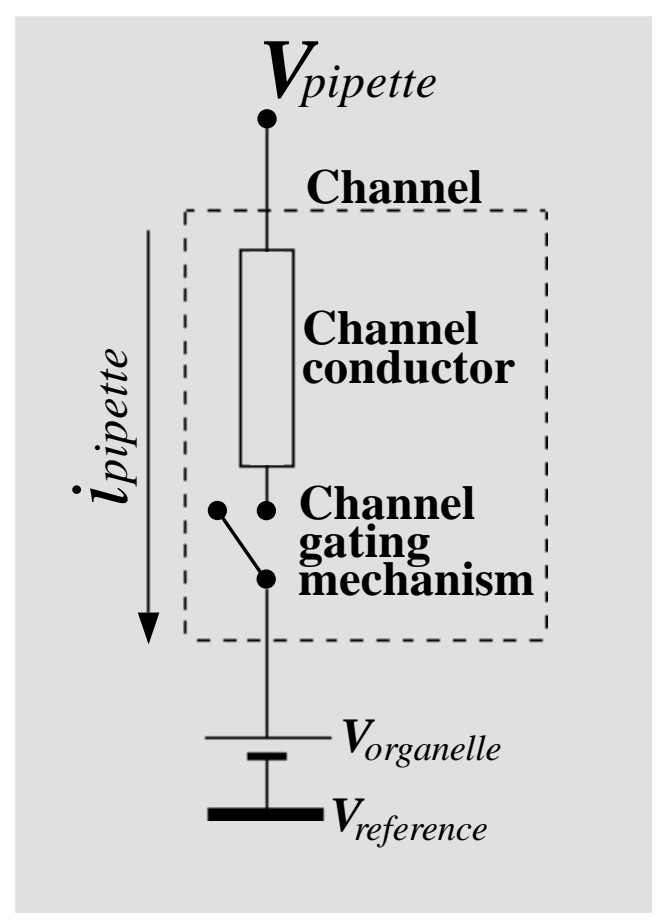

sential to the electrophysiological discussion that will follow. The space delimited by ONM and INM is a cisterna used by many processes to store proteins, molecules, etc. It is there that $\mathrm{Ca}^{2+}$ is thought to mediate NPCMMT (e.g., 11,12). Note that the outer membrane of the NE is contiguous with the rough endoplasmic reticulum. Therefore, the ONM and rough endoplasmic reticulum, although distinct structures, share many properties. This contiguity may cause arguments when it comes to identifying the source of ion channel activity. Therefore, it is not sufficient to simply place the patch-clamp pipette at the outside of the nucleus but one is required to prove that the activity is not derived from the rough endoplasmic reticulum. In a recent paper, it was shown that the large-conductance ion channel activity recorded in cardiac myocyte nucleus-attached patches does not derive from the endoplasmic reticulum (13). That this was the case was demonstrated by the inefficacy of various maneuvers known to affect protein-conducting channels of the endoplasmic reticu$\operatorname{lum}(14)$.

\section{The patent diameter of the NPC}

Many electron and fluorescence microscopy studies indicate that small molecules, ions and particles placed on one side of the NE reach the other side (e.g., 7,15,16). These observations have been interpreted to mean that the NPCs have a 'patent' diameter (a sort of effective cross-section for the electrolyte-filled hole) of about $10 \mathrm{~nm}$. The value of the patent diameter can vary from a few to several tens of nanometers according to the cell type and cycle (e.g., 7,16). This large patent diameter leads to the inescapable conclusion that monoatomic ions flow freely along the NPCs and, consequently, that any observed heterogeneous concentration distribution of small ions and molecules must result from 'cytoplasmic exclusion' (17) caused by the compartmentalized distribu- 
tion of water- and molecular-binding sites (e.g., 7,15,18) from another (or more) nonNE mechanism(s), or from technical artifacts. The 'cytoplasmic exclusion' principle states that because the state of water is different in the different cellular compartments, the solubility, and thus the concentration, of a solute is compartmentalized (in our case, between the nucleus and cytoplasm). This exclusion principle resembles the 'association-induction hypothesis' introduced at the same time to explain the resting potential of cells without the need for a plasmalemmal Na-K pump (e.g., 19,20). A discussion of nuclear resting potentials is outside the scope of this review.

\section{Agreement between patch-clamp and non-electrophysiological data}

The patent diameter view of unrestricted flow of ions along the NPCC is in apparent contradiction with the electrophysiological data indicating that the NPC behaves as an ion channel (e.g., 13,21-24). However, this discrepancy is eliminated when both qualitative and quantitative analyses are performed (15). Qualitatively, at the present time only the patch-clamp is capable of detecting live single NPC conformational changes through the measurement of NPCC ion-conductive properties. Not even the most sophisticated electron or light microscope techniques are capable of giving a measurement of the live function of a single NPC and, although atomic force microscopy and other scanning probe microscopies hold this promise, reports have yet to appear with the time-resolution required. All we can say with any kind of microscopy is that the particles have moved from one side to the other within an approximate time interval of minutes, whereas with the patch-clamp we can have time resolution down to the millisecond range or even less. Thus, we can say that $10-\mathrm{kDa}$ dextran molecules have passed from the outside of the nucleus to the inside within, say, $15 \mathrm{~min}$, but, in physical terms, this does not mean that the NPCC is permanently open as commonly concluded. What patch-clamp measurements show is that the NPC gates behave like any other gate controlling the traffic of large quantities. Quantitatively, one may neglect particle-particle and particle-NPCC wall interactions, and simply use the geometrical parameters reported for the NPCC to estimate the single channel conductance of the NPCC, $\gamma_{N P C C}$. Table 1 gives the values of $\gamma_{N P C C}$ assuming that the hydrated radius, $a_{i o n}$, of the current carrying ions (e.g., $\mathrm{K}^{+}$) is 0.2 $\mathrm{nm}$ (15), and that the NPC has a hole of 3-10$\mathrm{nm}$ radius, $r_{N P C C}, 25-100-\mathrm{nm}$ length, $l_{N P C C}$, (to accommodate published data; see 8,15) and filled with an electric conductor (the electrolyte) of specific resistivity, $\rho$, of 100$250 \Omega . \mathrm{cm}$ (around that of cytoplasm and nucleoplasm, plus or minus small inert contaminants, not medium-sized or larger macromolecules). The values are calculated when the resistance of each NPCC, $R_{N P C C}$, is approximated by the following equation (15):

$$
\begin{aligned}
& R_{N P C C}=\left\{\rho\left[l_{N P C C}+\left(1.64 r_{N P C C}\right)\right]\right\} /\left\{\pi\left(r_{N P C C}\right)^{2}\right. \\
& \left.\left[1-\left(a_{i o n} / r_{N P C C}\right)\right]^{6}\right\}
\end{aligned}
$$

Table 1 shows examples of $R_{N P C C}$ values calculated with equation 10 and covers published values (25-29). In consideration of the NPC variation with cell type and cell cycle phase as well as the measurement errors (instrument, sample preparation, etc.), we can say that there is good agreement between electrophysiological and non-electro-

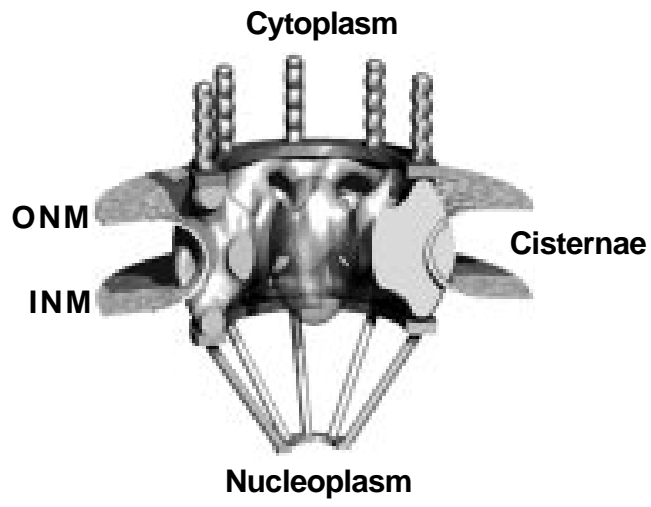

Figure 2 - 3D-Reconstruction model of the nuclear pore complex (NPC) generated from electron microscopy data. Cyto- and nucleoplasmic filaments are shown pointing to their respective compartments. The outer and inner nuclear membranes (ONM and INM, respectively) of the nuclear envelope (NE) create a space which serves as cisternae for the storage of important factors, one of which is $\mathrm{Ca}^{2+}$. Note that the putative central plug is shaded to indicate the uncertain nature of the concept. (From Ref. 5, with permission). 


\begin{tabular}{|c|c|c|c|c|c|c|}
\hline $\begin{array}{l}\text { Parameter } \\
\text { changed }\end{array}$ & $\begin{array}{c}\gamma_{N P C C} \\
(\mathrm{pS})\end{array}$ & $\begin{array}{c}R_{N P C C} \\
(\mathrm{G} \Omega)\end{array}$ & $\begin{array}{c}\rho \\
(\Omega . \mathrm{cm})\end{array}$ & $\begin{array}{l}I_{N P C C} \\
(\mathrm{~nm})\end{array}$ & $\begin{array}{c}r_{N P C C} \\
(\mathrm{~nm})\end{array}$ & $\begin{array}{l}a_{\text {ion }} \\
(\mathrm{nm})\end{array}$ \\
\hline INPCC & 451 & 2.2 & 100 & 100 & 4.5 & 0.2 \\
\hline$I_{N P C C}$ & 844 & 1.2 & 100 & 50 & 4.5 & 0.2 \\
\hline$I_{N P C C}$ & 1496 & 0.7 & 100 & 25 & 4.5 & 0.2 \\
\hline$r_{N P C C}$ & 1852 & 0.5 & 100 & 25 & 5 & 0.2 \\
\hline$r_{N P C C}$ & 1171 & 0.9 & 100 & 25 & 4 & 0.2 \\
\hline$r_{N P C C}$ & 625 & 1.6 & 100 & 25 & 3 & 0.2 \\
\hline$\rho$ & 416 & 2.4 & 150 & 25 & 3 & 0.2 \\
\hline$\rho$ & 312 & 3.2 & 200 & 25 & 3 & 0.2 \\
\hline$\rho$ & 250 & 4.0 & 250 & 25 & 3 & 0.2 \\
\hline
\end{tabular}

physiological data. For example, avian erythrocytes display a channel activity with a cationic channel conductance of $800 \mathrm{pS}$ (23), coconut endosperm cells with one of about $1,000 \mathrm{pS}$ (30), and murine cardiac myocytes show conductance values between 106 and $532 \mathrm{pS}\left(22-36^{\circ} \mathrm{C}\right)$, with higher values corresponding to temperatures closer to that of this warm-blooded species (31). This temperature dependence of $\gamma_{N P C C}$ demonstrates the complexity of the biochemical processes involved in NPCC gating (e.g., ATPases and GTPases). Reports of nuclear ion channels which could be attributed to NPCCs have been published (e.g., 23,24,30,32). However, these did not include demonstrations that the NPCCs were either functional or permanently closed, blocked or plugged. Other ion channels which are putatively notNPCCs have also appeared. These conclude that the recorded channel activity can be attributed to $\mathrm{Ca}^{2+}$-activated $\mathrm{K}^{+}$channels (33; see also 34$), \mathrm{IP}_{3}$-sensitive $\mathrm{Ca}^{2+}$-channels (3537; see also 34) and even now the $\mathrm{Cl}^{-}$channel known as the cystic fibrosis transmembrane conductance regulator or CFTR (38). Despite the assertion by the authors that these channels were not NPCCs, no proof was given that the NPCs were closed or plugged. We will discuss the importance of providing such proof in the following paragraphs (see section Identification of NPCs as a source of ion channel activity).

\section{Patch-clamp principles}

The patch-clamp demonstration that the NPCC opens probabilistically (13) is consistent with non-electrophysiological observations. Briefly, the NPCCs switch between open and closed states with open and close probabilities ( $p_{o}$ and $p_{c}$, respectively). These probabilities are regulated by cytosolic and nucleosolic factors such as GTPases, and localization signal receptors (e.g., 13,25,26). Therefore, despite the large diameter of the NPCC, the time average of its opening is less than the geometrical diameter of the NPC hole (what we are calling here the NPCC) unless, of course, $p_{o}$ is 1 . As a corollary, conditions that favor the closed state will reduce the average nucleocytoplasmic ion flow. The more the NPCC dwells in either the closed or the plugged state (where ion flow/current is zero), the lower the $p_{o}$ and the smaller the macroscopic ion current. A reduced macroscopic NE ion current favors the build-up of nucleocytoplasmic gradients of small ions, molecules and particles. Such generation of nucleocytoplasmic gradients (e.g., during $\mathrm{Ca}^{2+}$ transients, see 39) lend themselves to be misinterpreted as a reduced diameter of the NPCC but, instead, they are a reflection of the lower than unity open probability (i.e., $p_{o}<1$ ).

Early patch-clamp investigations suggested that ion channel activity recorded from the NE was attributable to NPCs (e.g., 23,24,30-32,40). In pure saline solutions containing high $\left[\mathrm{K}^{+}\right]$, these NPCC candidates have a linear current-voltage relationship and, therefore, they have constant single channel conductance, $\gamma_{N P C C}$ (e.g., 23,24,30$32,40)$. To date, only the large-conductance cardiac channel activity has been directly demonstrated to derive from NPCs (13; see below). Like some of the NE channels in other nuclear preparations, these cardiac channels show a preference for cations (40). Cardiac myocyte NPCCs display two modes of operation labeled 'stationary' and 'inacti- 
vating' (31). This behavior has been observed also in hepatocytes (41). In the 'stationary' or steady-state mode, the NPCCs open and close with a binomial behavior similar to an electron spin ensemble. That is, not only do all the NPCCs appear to be identical but they also switch between the open and closed states with open probabilities which are well fitted by a binomial model:

$P_{o}(n)=N ! p_{o}{ }^{n}\left(1-p_{o}\right) N-n /[n !(N-n)]$ (Eq. 11)

where $P_{o}(n)$ is the probability of finding $n$ channels simultaneously open, $p_{o}$ is, as before, the single channel open probability, and $N$ is the total number of ion-conducting channels in the patch (31). Note that equation 2 does not contain reference to time. This is because the system is in steady state and its stochastic characteristics are the same at any given time. Also note that, as indicated at the beginning of this review, for a perfect binomial system, $p_{o}+p_{c}=1$. In the 'inactivating' mode of operation, NPCCs respond to voltage stimulation (equivalent to electrochemical gradient stimulation; see Ref. 42) with an exponential decay in $P_{o}(n)$. That is, $P_{o}(n)$ is time-dependent.

$P_{o}(n, t)=P_{o}(n, 0) \mathrm{e}^{-t / \tau}$

with $t$ being time - the independent variable, $\tau$ the decay time constant, and $P_{o}(n, 0)$ the initial value of $P_{o}$. Note that $P_{o}$ does not necessarily follow the binomial behavior described in equation 10 . The term inactivating was assigned to this mode of operation because there are instances where the channels can no longer open upon successive application of voltage pulses but require a time for recovery (31). This time, although not always the same, is equivalent to the refractory period of nerves associated with sodium channel inactivation (see 42). The lack of constancy of the refractory period may be indicative of the much greater complexity of the NPCC mechanism of operation. For example, GTPase may play an im- portant role in the inactivating machinery (see 40). Similarly to classical inactivation, the time constants for this putative inactivation are reduced with voltage (see 42 ). At voltages between $-10 \mathrm{mV}$ and $+10 \mathrm{mV}$, cardiac NPCCs showed no measurable inactivation: they were open all the time (31). This suggests that, at least in quiescent, non-stimulated cardiac myocytes (where the reversal potential is negligible), the NPCCs offer little opposition to ion flow. However, when the voltage gradient across the NE increased (i.e., increase in electrochemical gradient across the NE), the probability of finding open channels decreased. This dependence of NPCC gating on voltage (electrochemical gradient) is consistent with the fluorescence microscopy observation that the NE becomes a $\mathrm{Ca}^{2+}$-barrier when the cytosolic $\left[\mathrm{Ca}^{2+}\right]$ increases over $300 \mathrm{nM}$ (39). This can also be due to $\mathrm{Ca}^{2+}$-induced $\mathrm{Ca}^{2+}$ release from the NE cisternae which, in turn, leads to NPCC plugging by macromolecules (see below). Since live cardiac myocytes in situ are never at rest, it is likely that NPCCs play a significant role in determining the signals that cross to one or the other side of the NE.

Putative NPCC substates of 25-50 pS were reported for cardiac myocytes (31, 40,43). However, it is not clear whether these may represent NPCs whose holes have been partially occluded by medium-sized molecules (see below). Cardiac myocyte NPCC activity was enhanced by cAMP-dependent protein kinase (PKA) and reduced by a PKA inhibitor (31). It was also shown that their activity could be suppressed with GTP- $\gamma$ S, a non-hydrolyzing analog of GTP, suggesting a direct involvement of GTPase in NPCC gating (40). It is unclear how this phenomenon correlates with the essential role that the small GTPase Ran/TC4 plays in MMT (e.g., 2-6,44). The current availability of methods for the isolation of NPCs (e.g., 45) should make it possible in the future to dissect the single NPCC gating characteristics in artificial lipid bilayer systems. 
Basic concepts in the identification of NPCs as a source of ion channel activity

The idea that NE ion channel activity derives from NPCCs is very appealing from the electrophysiological point of view and, indeed, efforts have been made towards demonstrating that this activity derives from NPCCs (e.g., Innocenti and Mazzanti (46) used osmotic shock). However, patch-clamp investigations complemented with laser-scanning confocal fluorescence microscopy, transmission electron microscopy, field-emission scanning electron microscopy, and atomic force microscopy showed a direct correlation between the large-conductance ion channel activity recorded from cardiac myocytes and myocyte NPCs (13). Both in the classical microelectrode studies of Loewenstein and coworkers (reviewed in 47) and in the first patch-clamp investigations of NE ion channel activity $(23,24)$, it was proposed that the NPC was the structure underlying the recorded activity. However, this proposition was hindered by patch-clamp investigations indicating that ion channels different from NPCCs also exist in either of the two membranes delimiting the NE. Such is the case for standard and CFTR chloride channels $(38,48)$, for $\mathrm{Ca}^{2+}$-sensitive $\mathrm{K}^{+}$channels (33), for $\mathrm{Ca}^{2+}$ - and $\mathrm{Zn}^{2+}$-channels (49, see 34), $\mathrm{IP}_{3}$-sensitive $\mathrm{Ca}^{2+}$-channels (34-37), etc. (50). That non-NPCCs do exist at the NE is further suggested by the demonstration that ion channel-forming proteins have been isolated from NE preparations (e.g., 34,51). Indeed, even a chloride ion channel protein which localizes primarily in the nucleus has been cloned and characterized (e.g., 52). Despite the non-NPCCs identified or yet to be identified at the NE, the NPCCs continue to stand out as the sole structures providing the direct pathway for the exchange of material between the nuclear and cytoplasmic compartments. For this reason, when the patch-clamp technique is used in the nucleusattached mode (i.e., the pipette tip forming a gigaseal with the outer membrane of the $\mathrm{NE}$ ), and when it is demonstrated that small and medium-sized (e.g., $<10 \mathrm{kDa}$ ) molecules enter the nucleus, then it must be unavoidably concluded that the NPCCs are effective electrical shunts for any non-NPCCs between the recording patch-clamp pipette and bath electrodes (note that all non-NPPCs are located on either membrane of the NE whereas the NPCCs join the two membranes). Therefore, from an electrophysiological point of view, NPCCs should be the natural candidates for the source of large conductance channel activity. Figure 3 aims at explaining these concepts with the equivalent circuit. For the sake of clarity, we will only discuss the nucleus-attached patch-clamp mode of recording. Figure 3 summarizes the three groups of channels found in the NE preparation. They are the NPCCs and the outer and the inner nuclear membrane channels (ONMC, and INMC, respectively). In nucleus-attached patches, the ONM and the INM can be thought of as two membranes in series. Since the pipette tip only touches the ONM, only the pipette voltage $\left(V_{\text {pipette }}\right)$ is applied to the NPCCs and the ONMCs. The voltage of the NE cisternae $\left(V_{N E-\text { cisternae }}\right)$ does not change appreciably and, consequently, there is no voltage drop across the INM and the INMCs. The NPCCs and ONMCs form a system of parallel conductors through which the measured pipette current $\left(i_{\text {pipette }}\right)$ is distributed into essentially two branches: the NPCC current $\left(i_{N P C C}\right)$ and the ONMC current $\left(i_{O N M C}\right)$. That is: $i_{\text {pipette }}=i_{N P C C}+i_{O N M C}$. Each current component is determined by the value of the conductance of the corresponding channel and the voltage applied to the channel. In cardiac myocytes, $V_{\text {nucleus }}$ is negligible in buffered $150 \mathrm{mM} \mathrm{KCl} \mathrm{(31).}$ That is, the nuclear interior is short-circuited to the bath. Thus, $V_{\text {nucleus }}$ equals the reference or bath voltage $\left(V_{\text {reference }}\right)$. For this reason, the voltage sensed by the NPCCs and the ONMCs equals $V_{\text {pipette }}$.

Since the density of NPCs is relatively 
high (e.g., 7,16), the patch-clamp pipette always contains a few or more NPCCs. Furthermore, because inside the patch there are many NPCCs with $\gamma_{N P C C}$ of several hundreds of $\mathrm{pS}$, it is clear that NPCCs are the major contributors to the recorded ion channel activity. Therefore, the only available explanation against the NPCCs being responsible for the observed ion channel activity is that the NPCCs are permanently closed (or plugged) due to the conditions used in patchclamp studies (e.g., saline not supplemented with MMT substrates). This counter-argument remains to be proven. Furthermore, fluorescence microscopy data clearly demonstrate that under saline conditions that prevent MMT, the small monoatomic ions and molecules diffuse freely between the nucleus and cytosol (e.g., 53). That is, even in the most unfavorable conditions for MMT, the NPCs still allow ion transport. Therefore, a corollary of this discussion is that any report claiming non-NPCC activity should be accompanied by a direct demonstration that the NPCCs are either closed, plugged or both.

\section{Peripheral channels of the NPC}

Only one computer-assisted 3-D reconstruction work, based on electron microscopy data, shows that the eight-fold geometry of the NPC contains eight parallel pathways for the transport of ions which are independent of the large central channel of the NPC (54). If these pathways behaved like ion channels, then one should see tens to hundreds of channels in a single nucleusattached patch because the NPC density per unit area ranges from a few NPCs to tens of NPCs per square micrometer or several channels with identical substates. However, this observation has never been reported and the 3 -D reconstruction work has not been duplicated. Nevertheless, it should be noted that efforts to find a large-conductance ion channel which shows eight substates have been recently published (41). Therefore, the only
$\mathbf{A}$

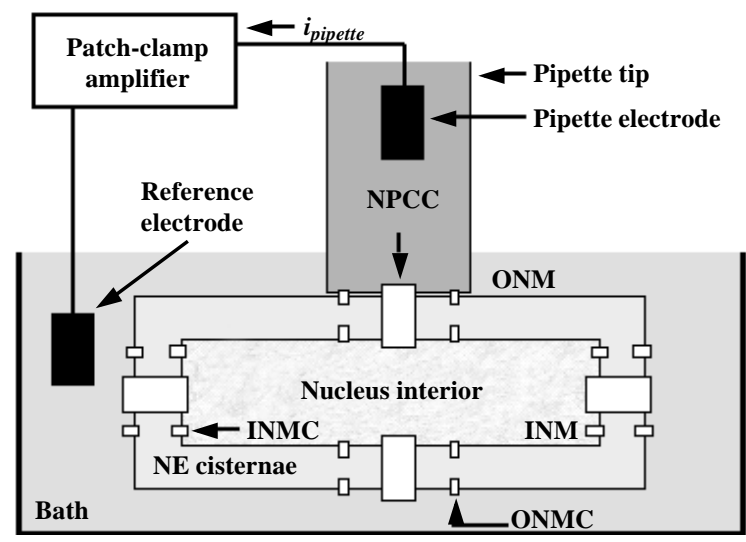

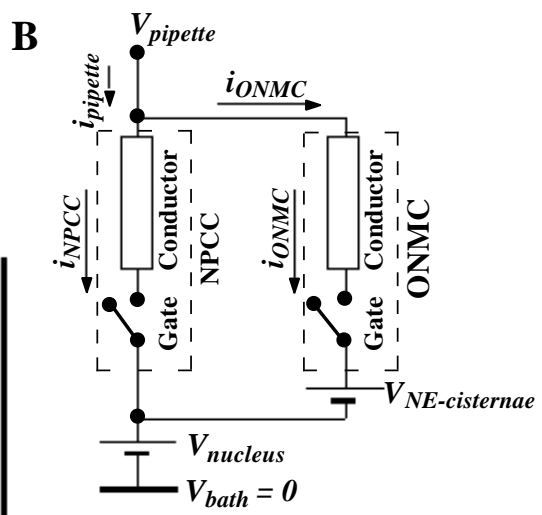

Figure 3 - Equivalent circuit for the ion channels at the nuclear envelope (NE). $A$, Schematics of the nucleusattached patch-clamp pipette system. B, Equivalent circuit. Three groups of channels are represented: NPC channels (NPCC), outer nuclear membrane channels (ONMC), and inner nuclear membrane channels (INMC). Under nucleus-attached patch-clamp configuration, the NPCCs and ONMCs form a system of parallel conductors through which the measured pipette current $\left(i_{\text {pipette }}\right)$ is distributed: the NPCC current $\left(i_{N P C C}\right)$ and the ONMC current ( $\left.i_{\text {ONMC }}\right)$. There is no contribution of INMC current $\left(i_{\text {INMC }}\right)$. That is: $i_{\text {pipette }}=i_{\text {NPCC }}+i_{\text {ONMC }}$. Each current component is determined by the value of the conductance of the corresponding channel and the voltage applied to the channel. The applied voltage for NPCCs equals the difference between the pipette and nucleus voltages $\left(V_{\text {pipette }}-V_{\text {nucleus }}\right)$. The applied voltage for ONMCs and INMCs is $\left(V_{\text {pipette }}-V_{N E \text {-cisternae }}\right)$ and $\left(V_{N E \text {-cisternae }}\right.$ $V_{\text {nucleus }}$ ), respectively, where $V_{N E \text {-cisternae }}$ is the voltage at the NE cisternae. That large inert particles (e.g., colloidal gold and spherical dendrimers) go into the nuclear interior but not into the NE cisternae which indicates that the ONMC conductance is much smaller than $\gamma_{N P C C} . V_{\text {rev }}$ is assumed negligible. 
remaining possibility is that these pathways are not ion channels in the electrophysiological sense but, instead, permanently open peripheral holes as reported in the electron microscopy studies (54). However, if this were the case then the baseline current in patch-clamp recordings should be much greater than the zero value reported for all patch-clamp work. A simple computation demonstrates this fact:

$i_{\text {pipette }}=i_{N P C C}+i_{\text {peripheral }}=\gamma_{N P C C} V_{\text {pipette }}+$ $8 \gamma_{\text {peripheral }} V_{\text {pipette }}$ (Eq. 13)

where the measured pipette current equals the sum of the current through the NPCC and the current through the peripheral channels (indicated by the subscripts). $\gamma_{N P C C}$ and $\gamma_{p e-}$ ripheral are the conductance values for the NPCC and one peripheral channel, respectively. Figure 4 shows the equivalent circuit for this concept. The measured pipette current, $i_{\text {pipette }}$, is the sum of the currents flowing through the central and peripheral channels. That is, $i_{\text {pipette }}=i_{N P C C}+N i_{\text {peripheral }}$, where $N$ is the number of peripheral channels ( 8 in most cases, see Ref. 8). The putative peripheral channels should cause a baseline current to be recorded. However, patch-clamp investi-

Figure 4 - Equivalent circuit for the proposed NPC system formed by the central and peripheral channels. The measured pipette current, $i_{\text {pipette}}$, is split into the current for the central and peripheral channels $\left(i_{N P C C}\right.$ and $i_{\text {peripheral, }}$ respectively). That is, $i_{\text {pipette }}=i_{N P C C}+N i_{\text {peripheral }}$, where $N$ is the number of peripheral channels ( 8 most of the times, see Ref. 8). The peripheral channels should cause a baseline current to be recorded in nucleus-attached experiments. However, this is yet to be reported, suggesting that the peripheral channels have no access to the cytosol (i.e., the side where the pipette is attached). $V_{\text {rev }}$ is assumed to be negligible.

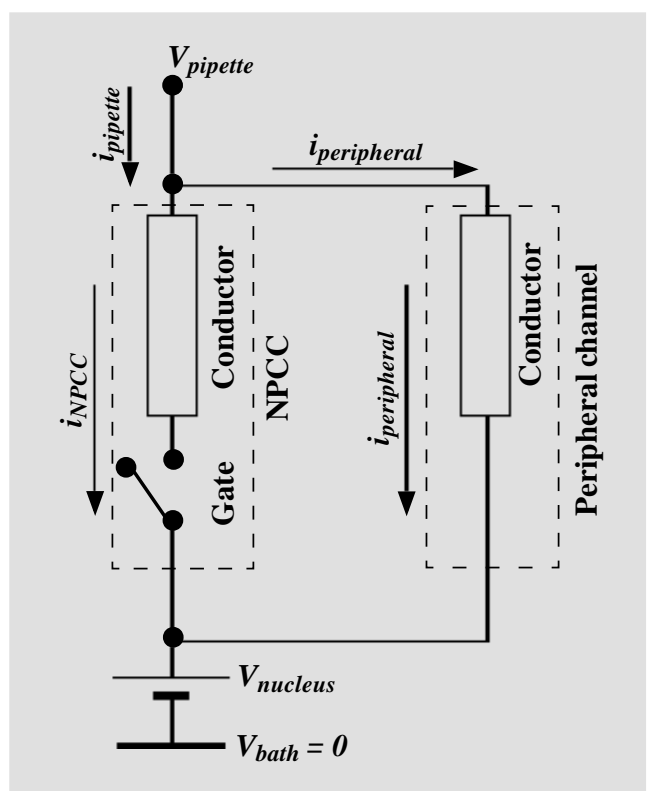

gations have reported no such current, suggesting that the peripheral channels do not have access to the cytosol.

\section{Technical issues}

A question commonly asked is whether it is possible to attain a gigaseal in preparations that have functional NPCs. In other words, do open NPCCs prevent gigaseal formation? The answer is no, they do not. An essential condition that must be technically attained prior to patch-clamp recording is the formation of the tight seal between the pipette tip and the membrane surface (in our case, the outer NE membrane). This seal has (or must have for a successful recording to be made) an electrical resistance, $R_{\text {seal }}$, greater than $1 \mathrm{G} \Omega$ and, for this reason, it is called the gigaseal. The gigaseal is assessed indirectly by reading the current flowing through the pipette, $i_{\text {pipette }}$, elicited with small current pulses (resulting from the application of small voltage pulses to the pipette electrode). If the membrane contains many open NPCCs, it is likely that the investigator may think that a gigaseal has not been reached. However, this interpretation is erroneous. Say that under the pipette tip there are 10 permanently open NPCCs and that their single channel conductance, $\gamma_{N P C C}$, is $500 \mathrm{pS}$. This would cause the pipette tip to see a patch conductance not lower than $5,000 \mathrm{pS}$ or $5 \mathrm{nS}$. This is equivalent to $0.2 \mathrm{G} \Omega$. The investigator could mistakenly take this to suggest that a gigaseal has not been formed. However, the fact is that the seal resistance between the rim of the pipette tip and the outer NE membrane, $R_{\text {seal }}$, cannot be measured directly with the patch-clamp technique. All that can be said is that the measured resistance, $R_{\text {measured }}$, is not greater than that of either the NE patch directly under the pipette tip, $R_{\text {patch }}$, or the seal resistance, $R_{\text {seal }}$. The relationship among these three quantities is:

$1 / R_{\text {measured }}=\left(1 / R_{\text {patch }}\right)+\left(1 / R_{\text {seal }}\right)$

(Eq. 14) 
Note that the contribution of other resistances (e.g., pipette, bath) are negligible and, thus, ignored in this discussion. Therefore, one can have a gigaseal and yet have a patch of NE containing many NPCs. The value of the gigaseal is revealed during moments of simultaneous NPCC closure observed during prolonged periods of recording (up to $72 \mathrm{~h}$ ), when the NPCs seem to exhaust the substrates for their gating mechanism (Bustamante JO, unpublished observations). Thus, the initial current recorded from a nucleus-attached patch may suggest that a gigaseal has not been reached and, consequently, the experimenter may discard the preparation. However, after many minutes of observation one may capture a simultaneous closure of the channels (Bustamante JO, unpublished results). Clearly, if the gigaseal had not been attained, this could be considered a poor pipette-membrane seal. To further confirm that the tight seal is retained, one may plug the NPCCs by adding diluted amounts of nuclear proteins (those which have nucleartargeting sequences) without transport substrates (i.e., all those substances that participate in the successful NPC-MMT: ATP, GTP, cytosolic and nucleosolic receptors, etc.). In this manner, it is possible to reduce the number of unplugged NPCCs and, therefore, to clearly differentiate and count the NPCCs in the NE patch (Bustamante $\mathrm{JO}$, unpublished results). It is also possible to obtain a gigaseal between the pipette tip and the NE but not see ion channel activity (Bustamante JO, unpublished observations). This, however, cannot be taken as indicative that the NPCs are not present or that they are not functioning, for if given a sufficient amount of time (15-60 min, depending on the availability of NPC-MMT substrates), NPCC activity will appear as a consequence of macromolecular unplugging. As discussed below, during translocation, macromolecules plug the NPCC for a period of time that will depend on the ex- perimental conditions. Since most patchclamp studies are carried out in pure saline solutions, macromolecular transport has the least favorable conditions. Therefore, those NPCCs plugged during nucleus isolation will remain that way for a very long time or forever, depending on the specific methodologies used. The longer the isolated nuclei are exposed to saline, the less transport substrates are left in the preparation and the greater the number of plugged channels (Bustamante JO, unpublished observations). This observation can be used to the investigator's advantage because it provides a means of controlling the number of unplugged, ion-conducting NPCCs. This is advantageous because for the analysis of single channel activity it is best to have as low a number of gating channels as possible (in the best case, only one). Therefore, with some fine tuning, a method can be developed that provides such a favorable situation for the statistical analysis of single channel activity (e.g., 13,31,40).

\section{Nucleocytoplasmic gradients}

We indicated above that electrophysiological and non-electrophysiological data are not adversaries (see also Table 1) but, does NPCC activity explain nucleocytoplasmic gradients of small ions and other molecules? On the basis of NPCC activity alone (i.e., $p_{o}, \gamma_{N P C C}$ and $N$ ), the amount of monoatomic ion flow exchanged per cardiac myocyte patch was estimated at leading to a concentration change of $1 \mathrm{mM} / \mathrm{s}$ (see Appendix in Ref. 31). Briefly, the maximal rate of change in ion concentration caused by the NPCC current in an NE patch is calculated by dividing the ionic current by the nuclear volume (e.g., about $500 \mu \mathrm{m}^{3}$ for a small spherical nucleus of $5 \mu \mathrm{m}$ radius, $\left.r_{\text {nucleus }}\right)$. For a patch with an average of $0.5 \mathrm{pA}$ in $1 \mathrm{~s}$ (Figure 1 in Ref. 31), the change in ion concentration per unit time was calculated at $1 \mathrm{mM} / \mathrm{s}$. Clearly, for the whole NE, the value 
would be several-fold greater. For example, for a pipette tip of $0.5 \mu \mathrm{m}$ radius, $r_{\text {pipette }}$, the minimal NE patch area (corresponding to a flat plane and not to an $\Omega$-shaped surface because no suction is applied) would be 0.8 $\mu \mathrm{m}^{2}$ or $\pi\left(r_{\text {pipette }}\right)^{2}$. The whole NE surface for the 5- $\mu \mathrm{m}$ radius nucleus in our example has an area of $314 \mu \mathrm{m}^{2}$ or $4 \pi\left(r_{\text {nucleus }}\right)^{2}$. Therefore, the estimated concentration change in 1 $\mathrm{s}$ for the whole NE is $393 \mathrm{mM}$. This estimate demonstrates that NPCC behavior does not oppose the concept of diffusion, although the latter is usually assumed to indicate that the NPCs do not switch between open and closed states. Table 2 shows calculations for various changes in the parameters of the nucleus and NPCC.

\section{NPC-mediated macromolecular translocation}

Although it has been demonstrated by fluorescence microscopy that nucleocytoplasmic ion flow is maintained even in isolated nuclei under pure saline conditions (e.g., 53), one arguable flaw in most patchclamp studies is the lack of simultaneous tests for the basic transport properties of NPCs. In those studies favoring NPCs as the source for channel activity, there is no visual demonstration of translocation. Whereas in those assuming that the activity derives from structures different from NPCs, there is absence of visual demonstration that the NPCs are closed. To resolve this problem, one can

\begin{tabular}{|c|c|c|c|c|c|c|c|c|c|c|c|c|c|}
\hline \multirow{3}{*}{$\begin{array}{l}\text { Parameter } \\
\text { changed }\end{array}$} & \multicolumn{5}{|c|}{ NPCC } & \multicolumn{8}{|c|}{ Nucleus } \\
\hline & \multirow{2}{*}{$\begin{array}{l}\gamma_{N P C C} \\
(p S)\end{array}$} & \multirow{2}{*}{$\begin{array}{l}V_{N E} \\
(\mathrm{mV})\end{array}$} & \multirow[t]{2}{*}{$p_{\text {open }}$} & \multicolumn{2}{|c|}{$<$ Flow rate $>$} & \multirow{2}{*}{$\begin{array}{c}D_{N P C} \\
\left(\mathrm{NPC} / \mu \mathrm{m}^{2}\right)\end{array}$} & \multirow{2}{*}{$\begin{array}{c}r_{N} \\
(\mu \mathrm{m})\end{array}$} & \multirow{2}{*}{$\begin{array}{c}A_{N E} \\
\left(\mu \mathrm{m}^{2}\right)\end{array}$} & \multirow{2}{*}{$\begin{array}{l}N_{N P C} \\
\text { (Total) }\end{array}$} & \multicolumn{2}{|c|}{$V_{N}$} & \multirow{2}{*}{$\begin{array}{c}\Delta m_{\text {ion }} / \Delta t \\
(\mathrm{~mol} / \mathrm{s})\end{array}$} & \multirow{2}{*}{$\begin{array}{r}\Delta C_{\text {ion }} / \Delta \\
(\mathrm{mM} / \mathrm{s}\end{array}$} \\
\hline & & & & $(\mathrm{pA})$ & $(\mathrm{mol} / \mathrm{s})$ & & & & & $\left(\mu \mathrm{m}^{3}\right)$ & (l) & & \\
\hline$p_{\text {open }}$ & 500 & 10 & 1.00 & 5.00 & $5 \mathrm{E}-17$ & 10 & 5 & 314.2 & 3142 & 523.6 & $5 E-13$ & $1.5 \mathrm{E}-13$ & 300 \\
\hline$p_{\text {open }}$ & 500 & 10 & 0.50 & 2.50 & $2.5 \mathrm{E}-17$ & 10 & 5 & 314.2 & 3142 & 523.6 & $5 E-13$ & $7.9 \mathrm{E}-14$ & 150 \\
\hline popen & 500 & 10 & 0.25 & 1.25 & 1.3E-17 & 10 & 5 & 314.2 & 3142 & 523.6 & $5 \mathrm{E}-13$ & 4.0E-14 & 75 \\
\hline$p_{\text {open }}$ & 500 & 10 & 0.10 & 0.50 & $5.0 \mathrm{E}-18$ & 10 & 5 & 314.2 & 3142 & 523.6 & $5 \mathrm{E}-13$ & $1.6 \mathrm{E}-14$ & 30 \\
\hline$p_{\text {open }}$ & 500 & 10 & 0.05 & 0.25 & $2.5 \mathrm{E}-18$ & 10 & 5 & 314.2 & 3142 & 523.6 & $5 \mathrm{E}-13$ & $7.9 \mathrm{E}-15$ & 15 \\
\hline$p_{\text {open }}$ & 500 & 10 & 0.01 & 0.05 & $5.0 \mathrm{E}-19$ & 10 & 5 & 314.2 & 3142 & 523.6 & $5 E-13$ & $1.8 \mathrm{E}-15$ & 3 \\
\hline$\gamma_{N P C C}$ & 400 & 10 & 0.50 & 2.00 & $2.0 \mathrm{E}-17$ & 10 & 5 & 314.2 & 3142 & 523.6 & $5 E-13$ & $6.3 \mathrm{E}-14$ & 120 \\
\hline$\gamma_{N P C C}$ & 300 & 10 & 0.50 & 1.50 & $1.5 \mathrm{E}-17$ & 10 & 5 & 314.2 & 3142 & 523.6 & $5 \mathrm{E}-13$ & 4.7E-14 & 90 \\
\hline$\gamma_{N P C C}$ & 200 & 10 & 0.50 & 1.00 & $1.0 \mathrm{E}-17$ & 10 & 5 & 314.2 & 3142 & 523.6 & $5 E-13$ & 3.1E-14 & 60 \\
\hline$\gamma_{N P C C}$ & 100 & 10 & 0.50 & 0.50 & $5.0 \mathrm{E}-18$ & 10 & 5 & 314.2 & 3142 & 523.6 & $5 \mathrm{E}-13$ & 1.6E-14 & 30 \\
\hline$\gamma_{N P C C}$ & 50 & 10 & 0.50 & 0.25 & $2.5 \mathrm{E}-18$ & 10 & 5 & 314.2 & 3142 & 523.6 & $5 E-13$ & $7.9 \mathrm{E}-15$ & 15 \\
\hline$\gamma_{N P C C}$ & 25 & 10 & 0.50 & 0.13 & 1.3E-18 & 10 & 5 & 314.2 & 3142 & 523.6 & $5 \mathrm{E}-13$ & $3.9 \mathrm{E}-15$ & 8 \\
\hline$D_{N P C}$ & 500 & 10 & 0.50 & 2.50 & $2.5 \mathrm{E}-17$ & 5 & 5 & 314.2 & 1571 & 523.6 & $5 E-13$ & 3.9E-14 & 75 \\
\hline$D_{N P C}$ & 500 & 10 & 0.50 & 2.50 & $2.5 \mathrm{E}-17$ & 3 & 5 & 314.2 & 942 & 523.6 & $5 E-13$ & $2.4 \mathrm{E}-14$ & 45 \\
\hline$D_{N P C}$ & 500 & 10 & 0.50 & 2.50 & $2.5 \mathrm{E}-17$ & 1 & 5 & 314.2 & 314 & 523.6 & $5 E-13$ & $7.9 \mathrm{E}-15$ & 15 \\
\hline$r_{N}$ & 500 & 10 & 0.50 & 2.50 & $2.5 \mathrm{E}-17$ & 10 & 10 & 1257 & 12566 & 4188.8 & $4 \mathrm{E}-12$ & $3.1 \mathrm{E}-13$ & 75 \\
\hline$r_{N}$ & 500 & 10 & 0.50 & 2.50 & $2.5 \mathrm{E}-17$ & 10 & 20 & 5027 & 50266 & 33510 & $3 E-11$ & 1.3E-12 & 38 \\
\hline$r_{N}$ & 500 & 10 & 0.50 & 2.50 & $2.5 \mathrm{E}-17$ & 10 & 30 & 11310 & 113098 & 113098 & $1 \mathrm{E}-10$ & $2.8 \mathrm{E}-12$ & 25 \\
\hline$r_{N}$ & 500 & 10 & 0.50 & 2.50 & $2.5 \mathrm{E}-17$ & 10 & 40 & 20106 & 201062 & 268083 & $3 E-10$ & $5.0 \mathrm{E}-12$ & 19 \\
\hline$r_{N}$ & 500 & 10 & 0.50 & 2.50 & $2.5 \mathrm{E}-17$ & 10 & 50 & 31416 & 314160 & 523600 & $5 \mathrm{E}-10$ & $7.9 \mathrm{E}-12$ & 15 \\
\hline
\end{tabular}


test the NPC ion transport properties with fluorescent probes (e.g., 53). Either 4-kDa FITC-dextran (Molecular Probes, Eugene, OR) or 5.4-nm FITC-labeled Starburst ${ }^{\mathrm{TM}}$ dendrimers (Polysciences, Warrington, PA) can be used (Bustamante JO, unpublished results). Starburst dendrimers appear a better choice as they have an almost perfect spherical shape (see Ref. 55). Laser-scanning confocal fluorescent microscopy measurements performed during electrophysiological investigations show that both $4-\mathrm{kDa}$ FITC-dextran and 5.4-nm FITC-dendrimers translocate under simple, high-K saline conditions (13; Bustamante JO, Geibel JP, Liepins A, Hanover JA and McDonnell TJ, and also Bustamante JO, unpublished results). This observation is consistent with the demonstration that $\leq 10-\mathrm{kDa}$ FITC-dextran molecules translocate across the NE under simple saline conditions that normally prevent MMT (53). These results demonstrate that the nuclei utilized in those electrophysiological studies conformed to the prevalent concept that NPCs allow translocation of not only monoatomic ions but also of small-to-medium-sized molecules and particles (53). More importantly, the nuclei utilized in one electrophysiological laboratory have been shown to retain their macromolecular transport capacity (13; Bustamante JO, Geibel JP, Liepins A, Hanover JA and McDonnell TJ, unpublished results). This capacity was demonstrated by constructing a nuclear protein probe with the fluorescent B-phycoerythrin (B-PE, $240 \mathrm{kDa}$ ) conjugated to the nuclear localization signal (NLS) of the SV40 large T antigen (13). Readers interested in the details of the mechanisms required for NPC-MMT are encouraged to consult excellent reviews which have appeared elsewhere (e.g., 2-6,45). For this reason, we focus here on the highlights of MMT. Briefly, various mechanisms have been identified for several classes of molecules. For proteins, the molecules carrying the NLS must first bind to a cytosolic receptor, then bind to a docking site at the NPC, and then translocate in an ATP-dependent process. The process depends on other factors, including Ran/TC4 and phosphorylation of some of the substrates involved. Note that the conjugation of B-PE to the NLS was required to make the complex recognizable by the NPC-mediated mechanism for MMT (13). Without conjugation to an NLS, B-PE will not enter the nucleus. Thus, the cardiac myocyte investigations demonstrated that ion channel activity was recorded in a preparation which retained the NPC capacity for ion and macromolecule transport. An advantage of conjugating a large fluorescent molecule to an NLS rather than conjugating a nuclear protein to a fluorescent probe such as FITC is that the large fluorescent probe will not enter the nucleus if not conjugated to the NLS whereas if the FITC conjugation of the nuclear protein is not strong enough, then the probe may enter the nucleus and give a false reading. Recent observations on changes of fluorescence response of fluorochromes caused by nuclear compartmentalization are important for absolute value determinations but not for relative, qualitative or semi-quantitative observations (56).

\section{The identification of NPCs as a source of ion channel activity}

Direct demonstration that the NPCs are the structure responsible for the open-close gating of large-conductance NE ion channels was given in isolated cardiac myocyte nuclei (nucleus-attached mode) by different tests (13). One of these showed the direct blockade of channel activity by the NPC monoclonal antibody mAb414 (known to block NPC-mediated macromolecular transport). Only this antibody, out of a series of NPC and NLS antibodies, did block the largeconductance ion channel activity (13). The lack of action was also seen with other nonNPC commercial antibodies (13). These nonNPC antibodies included anti-FITC antibody, 
anti-myosin, and the mAb414 isotype control (13). The blocking effect of mAb414 on channel activity was independently confirmed (57). The effect of mAb414 on NPCC activity parallels that seen in the mitochondrial multiple conductance channel upon the application of an antibody to a protein import component of the mitochondrial inner membrane (58). That mAb414 did not plug the NPCC, but instead blocked it, was shown by the unidirectional blockade (i.e., blockade seen with only voltage of one sign) seen in some preparations (13). The reader should note that this unidirectional blockade is more than a simple voltage-dependent blockade because $100 \%$ block occurred with only one voltage polarity. Taken together, the results support the conclusion that the NPC, like other organellar and junctional ion channels, has an intrinsic ion channel behavior with high conductance at high $\left[\mathrm{K}^{+}\right]$. That a supramolecular structure may have an intrinsic ion channel activity (switching between the open and closed states) and at the same time may account for a large ion permeability is exemplified by the many reports on organellar ion channels. For example, at these high $\left[\mathrm{K}^{+}\right]$ values, both the ryanodine receptor $\mathrm{Ca}^{2+}$ channel (reviewed in Ref. 59) and the mitochondrial multiple conductance channel (e.g., 60 ) have a single channel conductance of the order of $10^{3} \mathrm{pS}$. In analogy to other ion channels, NPCC activity depends on the voltage across the NE (and thus, the electrochemical potential). Note, however, that while the open probability decays faster (smaller time constants) at larger electrochemical potentials, the single channel conductance remains constant.

\section{Electrophysiological arguments favoring NPCs as ion channels}

In addition to these supportive data, one can use electrical circuit theory alone to deduce that in nucleus-attached patches of cardiac myocytes (with negligible resting poten- tial, $\left.V_{\text {nucleus }}\right)$ the NPCCs are the source for the recorded electrical activity. First, the hole of each NPC (i.e., the NPCC) allows a significant amount of ion flow and is solely responsible for the nucleocytoplasmic exchange of electrolytes (e.g., 8). Second, NPCCs have a wide diameter and are present in large numbers per unit area (e.g., 16). In cardiac myocytes, the number of NPCs per $\mu^{2}$ was estimated at 12 \pm 4 (see Ref. 13). Third, the NE outside the area covered by the pipette tip has negligible electrical resistance because it contains many ion-conducting NPCs. Therefore, the potential inside the nucleus is pretty much the same as that of the bath. Consequently, the potential sensed by the NPCs under the recording patchclamp pipette is given by the potential difference between the bath and pipette electrodes. Fourth, in nucleus-attached patches, where the pipette is sealed to the outer membrane of the $\mathrm{NE}$, only non-NPC channels on the outer NE membrane are directly affected by the changes in the pipette voltage (as typically occurs in the patch-clamp technique). Fifth, any ion current contribution from non-NPCCs $\left(I_{\text {other }}\right)$ at the ONM flows in parallel to current contributed by the NPCCs $\left(I_{N P C C}\right)$, i.e.,

$I_{\text {measured }}=\sum I_{N P C C}+\sum I_{\text {other }}$

where $\sum$ represents the sum total of the current contributed by all the channels of the corresponding class. Therefore, for the contribution of non-NPCCs to overcome that of NPCCs it would be necessary to have a higher density of channels per unit area and/ or a higher single channel conductance. As mentioned above, to date, this has not been shown. On the contrary, NPCCs have been shown to allow ion and small molecule translocation under pure saline conditions (53). Dextran molecules go into the nucleus but not into the NE (e.g., 53).

\section{Macromolecules nullify single NPC channel conductance}

Nuclear proteins, mRNA, native and for- 
eign nucleic acids and other macromolecules utilize the NPCs to cross the NE (e.g., 2-6). Since these macromolecules are poor electrical charge carriers and since they take over the volume previously occupied by the monoatomic ions (the good electrical charge carriers), one would expect NPC-MMT to reduce the ion conductance of a single NPCC, $\gamma_{N P C C}$. To date, only one laboratory has reported the effects of nucleocytoplasmic MMT on NPCC activity (e.g., 13,61,62). Nucleartargeted B-PE silenced the NPCC activity (13). However, the channel activity was progressively recovered with time, in a timecourse that resembled that required for the complete nuclear transport of the nucleartargeted B-PE probe in fluorescence microscopy experiments. It must, therefore, be concluded that while the channel activity was silent, the B-PE macromolecules were continuously transported into the nucleus. Experiments with transcription factors (an important group of naturally occurring nucleartargeted macromolecules involved in the regulation of gene activity) also transiently silenced ion channel activity $(13,61,62)$. These experiments demonstrated that when transcription factors (AP-1/c-Jun, NF- $\kappa \mathrm{B}$, SP1 and TATA-binding protein) and other nuclear-targeted molecules (e.g., B-PE conjugated to NLS) with a molecular mass of about $40 \mathrm{kDa}$ or higher were added, the ion channel activity was silenced for a period of time ranging from a few seconds (with substrates) to hours (without substrates). Both cases correlate with the electron microscopy observation that, without effective NPCMMT substrate (e.g., $\mathrm{Ca}^{2+}$, ATP, GTP, Ran/ TC4, cytosolic and nucleosolic receptors, etc.), the NPC is plugged (e.g., 8). Since transcription factors enter the nucleus exclusively through the NPCC, the conclusion followed that the recorded ion channel activity in these MMT-competent nuclei derived from gated ion flow along the NPC and not from an indirect action of the factors (13). When comparing the translocation times in these nuclei with the times in situ, the reader should be aware that the electrophysiological experiments were conducted under conditions which are far from optimal for normal macromolecular transport (e.g., 2-6). For example, cytosolic factors such as Ran/ TC4 and NLS receptors were not added. Furthermore, the rate of nuclear cytoplasmic protein transport has been shown to be determined by the protein phosphorylation (reviewed in Ref. 63). Therefore, it is likely that protein kinases and phosphatases play a role in nuclear transport by controlling the phosphorylation levels of both transport substrates and NPCs (see 63). Despite these limitations of the patch-clamp experiment, we consider these patch-clamp conditions (with less than optimal content of NPC-MMT substrates) to facilitate the isolation of single ion channel activity because under normal conditions the translocation should take place at a faster pace and might make it difficult to isolate the NPCC behavior. Furthermore, despite these non-physiological conditions, the preparations demonstrated an extraordinary stability, yet to be reported elsewhere (>72 h; 62; Bustamante JO, unpublished results). Therefore, we would like to note the remarkable (albeit difficult) success in patch-clamping the nucleus in situ (e.g., 64). This achievement should facilitate patch-clamp studies under more realistic conditions and make easier the comparison of patch-clamp results with those obtained in non-electrophysiological experiments (e.g., with permeabilized cells, see Ref. 11). Note, however, that filling the pipette with cytosol causes some electrical problems in patch-clamp recording as the pipette resistance increases (Bustamante JO, unpublished results).

The observation that macromolecules silence other large ion channels is not new. Indeed, the concept of a plug channel state distinct from the open, closed and inactivated states was proposed for other organellar preparations. For example, in mitochondrial outer membranes, a mitochondrial address- 
ing peptide silences a large conductance cationic channel (e.g., see 65 and references therein). This channel, known as the peptide-sensitive channel, is detected in mutant porin-less preparations (e.g., 66). The open probability of the voltage-dependent anion channel (VDAC) of the outer mitochondrial membrane (porin type-1; which is also expressed in sarcoplasmic reticulum; see Ref. 67) was eliminated with a soluble $54-\mathrm{kDa}$ mitochondrial protein (reviewed in Ref. 68). Mitochondrion-targeting peptides also plug the multiple conductance mitochondrial inner membrane channel whose properties resemble those of the peptide-sensitive mitochondrial channel (e.g., 58,60,69). Finally, protein translocation through the 4-6-nm diameter aqueous pore of the endoplasmic reticulum, the translocon, eliminated channel activity (e.g., 14,70). Independent fluorescence accessibility studies show that the aqueous pore in a functioning translocon is 4-6 $\mathrm{nm}$ in diameter, making it the largest membrane channel, after that of the NPC, that maintains a permeability barrier (71).

\section{NPC channel activity, MTT, and $\mathrm{Ca}^{2+}$ in $\mathrm{NE}$ cisternae}

It has recently been recognized that $\left[\mathrm{Ca}^{2+}\right]$ in the $\mathrm{NE}$ cisternae $\left(\left[\mathrm{Ca}^{2+}\right]_{\mathrm{NE}}\right)$ plays a major role in the NPC-mediated translocation of macromolecules (11) and medium-sized (>10 $\mathrm{kDa})$ molecules $(53,72$, reviewed in 12). Briefly, when $\left[\mathrm{Ca}^{2+}\right]_{\mathrm{NE}}$ is low, the translocation of macromolecules and even of medium-sized molecules $(>10 \mathrm{kDa})$ is not possible. It should, therefore, be expected that in cells where there is a great deal of $\mathrm{Ca}^{2+}$ release from the NE cisternae, the transport of macromolecules will be adversely affected. The impaired transport machinery, in turn, should cause the macromolecules to be caught in the middle of the NPC translocation hole (the NPCC). Therefore, like a plug, they should obstruct the pathway for ion flow and, consequently, result in an impaired translocation of small molecules and monoatomic ions. The period of silence must be determined by many factors. When the concentration of the macromolecules is sufficiently low, it may be possible to detect single translocations, whereas when it is relatively high, the many molecules may line up in single file to give the equivalent effect of translocation of a single long molecule. Translocation will stop when one of the required NPC-MMT substrates is exhausted (e.g., $\mathrm{Ca}^{2+}$, ATP, GTP, Ran/TC4, cytosolic and nucleosolic receptors, etc.), even if a macromolecule containing the signal is still present and ready for transport. That is, MMT cannot be expected to follow the customary diffusion laws. Therefore, rather than a process described by a single exponential change, a multi-exponential process should be expected due to the biochemical reactions involved (signal recognition, docking, etc.; see, e.g., 2-6). Thus, theoretically, each reaction (e.g., signal recognition) should correspond to an exponential function. Structural support for the electrophysiological idea of the translocating macromolecule behaving like a plug comes from the consistent and reproducible electron microscopy observation that when transport substrates are supplied, most NPCs are unplugged whereas when the substrates are absent, most of the NPCs are plugged (e.g., 8). Figure 2 shows the putative plug in the 3-D reconstruction NPC model of Pante and Aebi (8). The fuzzy, uncertain concept of the plug is indicated by the shaded coloring of the figure. That plugging material may account for a high NE resistance was put forward by Loewenstein and coworkers (22) more than three decades ago. Since both transcription factors and mRNA use the NPCs for their translocation, it can be concluded that the cellular rhythm affecting $\left[\mathrm{Ca}^{2+}\right]_{\mathrm{NE}}$ should be reflected on the levels of gene activity and expression.

A plethora of reports with fluorescence and luminescence microscopy support the concept of the NE as a barrier (e.g., 73-78; 
see 79 and 80). As discussed in previous paragraphs, each time the NPCC opens, it allows a great deal of ions to be translocated. Therefore, reports showing steady-state nucleocytoplasmic gradients may be explained by an NE that has many of its NPCCs plugged by translocating molecules or by the classical view of binding sites or water states (e.g., 15,18-20). Luminescence experiments with nucleoplasmin (a nuclear protein) fused to aequorin demonstrated the $\mathrm{Ca}^{2+}$ barrier nature of the NE $(73,74)$. However, when aequorin was fused to the nuclear localization signal of the glucocorticoid receptor, the nature of the NE as a barrier was not detected (81). This result may be related to simpler transport mechanisms for the receptor used for nuclear localization (e.g., 82). In other words, the selection of the protein to be used for nuclear targeting is critical to the interpretation of the results because the mechanisms of nuclear transport for proteins (e.g., nucleoplasmin) are not the same as those for steroid receptors (e.g., glucocorticoid receptor). The experimental data indicate the importance of macromolecular transport for the maintenance or development of nucleocytoplasmic gradients. This relationship between macromolecular transport and the ATP-independent flow of small molecules, particles and ions, should be relevant to the interpretation of experimental results. For example, when evaluating data from simultaneous fluorescence and 'whole-cell' patch-clamp recordings, the investigator should be aware that the pipette may dilute away transport substrates without which macromolecular transport would be impossible. This condition would lead to the NPCC being plugged by a macromolecule and, consequently, would result in an envelope with a full ion-barrier character. The reader should note that, due to the complexity of requirements for NPC-MMT, having a macromolecule with a nuclear localization signal plus ATP and $\mathrm{Ca}^{2+}$ is not in itself sufficient to secure translocation.
Changes in NPC topography concomitant with changes in NPCC activity were confirmed by atomic force microscopy (62). In these investigations, the universal transcription factor TATA-binding protein (with both structural and functional roles) facilitated transport and unplugging of the channels after the typical transient reduction in single NPCC conductance (62). The atomic force microscopy images suggested that the proteins docked and accumulated at the NPC cytoplasmic side (62). Part of the mechanisms of TATA-NPC interaction was attributed to an oligomerization effect mediated by protein glycosylation (62; see also 83 and references therein).

\section{Relevance to studies of gene control and expression}

The demonstration that the NPC has an intrinsic ion channel activity supports the use of the patch-clamp technique for the quantification of macromolecular transport along a single functional NPC. To date, no other technique has this capability. In analogy to the principles of the Coulter cell counters, basic electrophysiological principles show that, when completely filled with electrolytes, the NPCC ionic conductance is maximal $(13,84)$. They also show that, each time a macromolecule is translocated, the NPCC conductance goes to zero because the macromolecule displaces all the electrical charge carriers $(13,84)$. This phenomenon is not exclusive to the NPC but is shared by channels of mitochondria (e.g., $58,60,69)$ and endoplasmic reticulum (e.g., 14,70).

Perhaps the most dramatic example of this channel plugging phenomenon is the well-known NPC-mediated translocation of mRNA and ribonucleoprotein particles (e.g., $85)$ as well as foreign nucleic acids (e.g., 86,87). Figure 5 shows the basic mechanism for the translocation of the ribonucleoprotein particle (Figure 2 of reference 85; reproduced with permission). Not only do these 
Figure 5 - NPC-mediated ribonucleoprotein particle translocation. The particles change their geometry to traverse the NPCC. (From Ref. 82, with permission).
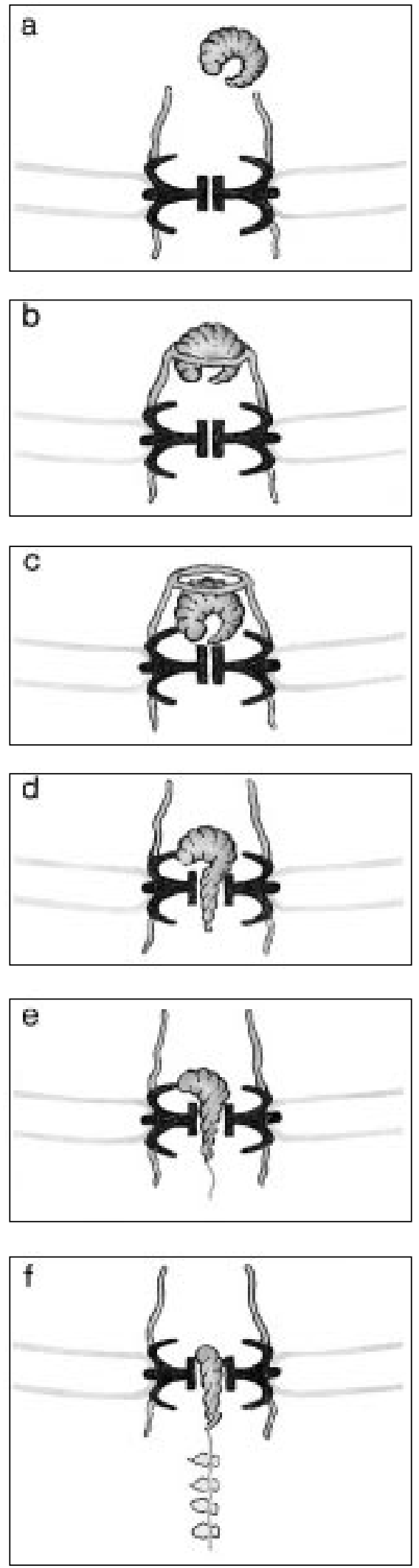

particles have wider diameter than the NPCC but their mechanism of translocation involves their transient transformation into filaments during their 'snaking' through the NPCCs (e.g., 86). Clearly, for smaller molecules which are poor charge carriers and do not plug the NPCC completely, the situation is intermediary and requires a more complex description. Indeed, experiments with 5.4$\mathrm{nm}$ FITC-labeled dendrimers showed that the probes translocate to the nucleus and during their translocation the binomial behavior of the NPCC is transformed into lowfrequency bursts (Bustamante JO, unpublished results). This subject is outside the scope of this review but, in principle, one can reason that for smaller molecules with poor charge carrier properties, the effect would be directly proportional to the density of the molecules inside the channel. The higher their density, the higher the resistivity of the electrolyte filling the NPPC and, therefore, the smaller the single channel conductance, $\gamma_{N P C C}$. A semi-quantitative analysis demonstrates the point. Under pure saline condition, when the NPC hole is completely filled with the electrolyte, the electrical charge density per unit time $\left(\rho_{\text {charge }}\right)$ is:

$\rho_{\text {charge }}=n_{\text {carrier }} / v_{N P C C}$

where $n_{\text {carrier }}$ is the number of good electrical charge carriers inside the NPC hole and $v_{N P C C}$, the volume of the hole. When macromolecules and other large, inert, non-currentcarrying particles enter the hole, they displace the charge carriers, thus reducing $n_{\text {carrier }}$ and thus, $\rho_{\text {charge }}$.

$v_{\text {carrier }}=v_{N P C C}-v_{\text {inert }}$

where $v_{\text {carrier }}$ and $v_{\text {inert }}$ are the volume occupied by carrier and inert particles, respectively.

$n_{\text {carrier }}=v_{\text {carrier }}\left[X_{\text {carrier }}\right]=\left(v_{N P C C}-v_{\text {inert }}\right)\left[X_{\text {carrier }}\right]$ (Eq. 18)

where $\left[X_{\text {carrier }}\right]$ is the concentration of elec- 
trical charge carriers. Thus,

$\rho_{\text {charge }}=\left(v_{N P C C}-v_{\text {inert }}\right)\left[X_{\text {carrier }}\right] / v_{N P C C}=$

$\left[1-\left(v_{\text {inert }} / v_{N P C C}\right)\right]\left[X_{\text {carrier }}\right]$

(Eq. 19)

$v_{\text {inert }}$ can be calculated from the concentration of electrically inert particles, $\left[X_{\text {inert }}\right]$, and their individual (or average) volume, $v_{\text {particle }}$ :

$v_{\text {inert }}=n_{\text {inert }} v_{\text {particle }}=v_{\text {NPCC }}\left[X_{\text {inert }}\right] v_{\text {particle }}$

Therefore,

$\rho_{\text {charge }}=\left(1-v_{\text {particle }}\left[X_{\text {inert }}\right]\right)\left[X_{\text {carrier }}\right]$

This relationship may be expanded to incorporate the presence of multiple charge carriers (e.g., $\mathrm{K}^{+}, \mathrm{Na}^{+}$and $\mathrm{Ca}^{2+}$ ) and multiple poor or inert carriers (e.g., transcription factors and nuclear proteins). The above analysis does not take into account interactions among particles or between the particles and the NPCC inner wall lining, which affect carrier mobility. In the real world, these are a significant factor opposed to free ion flow.

As we come to the end of this review, we would like to summarize by saying that both data and theory about the cell nucleus and other cellular organelles show that the period of channel silence can be correlated with the translocation time of macromolecules. This principle, denoted the 'macromolecule-conducting ion channel paradigm' in the study of NPC ion channel behavior (13), is similar to that of Coulter cell counters. The principle has been applied to polymer counting along ion channels (84) and to the determination of the size of nucleic acids with 2.6-nm diameter channels (88). The use of molecules which are not electrical charge carriers for the measurement of ion channel diameter has been applied to the determination of the diameter (ca. $3 \mathrm{~nm}$ ) of a porin channel (89). The observation that the patchclamp technique can be used to measure and study NPC-MMT for as long as $72 \mathrm{~h}$ (62; Bustamante JO, unpublished observations) implies that the mechanisms of transcription factor and mRNA translocation can be studied by this electrophysiological technique. Therefore, the possibility of using the patchclamp technique for the identification of the NPC mechanisms underlying transcription factor control of gene activity and of mRNAmediated gene expression offers a unique advantage over other currently available techniques in that it facilitates quantitative studies of macromolecular transport at the single NPC level. By complementing this technique with low light-level fluorescence microscopy, it should be possible to resolve many of the current enigmas pervading the field.

Further information related to this section can be found at the following internet site: http://www.geocities.com/TheTropics/ 6066/index.html.

\section{Acknowledgments}

The authors acknowledge the constructive criticisms of Alan Finkelstein, Michael V.L. Bennett, Peter Satir and Renato Rozental (Albert Einstein College of Medicine). 


\section{References}

1. Sakmann B \& Neher E (1995). SingleChannel Recording. Plenum Press, New York.

2. Gorlich D (1997). Nuclear protein import. Current Opinion in Cell Biology, 9: 412419.

3. Lee MS \& Silver PA (1997). RNA movement between the nucleus and the cytoplasm. Current Opinion in Genetics and Development, 7: 212-219.

4. Moroianu J (1997). Molecular mechanisms of nuclear protein transport. Critical Reviews in Eukaryotic Gene Expression, 7:61-72.

5. Nakielny S \& Dreyfuss G (1997). Nuclear export of proteins and RNAs. Current Opinion in Cell Biology, 9: 420-429.

6. Nigg EA (1997). Nucleocytoplasmic transport: signals, mechanisms and regulation. Nature, 386: 779-787.

7. Maul G (1977). The nuclear and the cytoplasmic pore complex: structure, dynamics, distribution, and evolution. International Reviews of Cytology, S6: 75-186.

8. Pante N \& Aebi U (1996). Molecular dissection of the nuclear pore complex. Critical Reviews in Biochemistry and Molecular Biology, 31: 153-199.

9. Rutherford SA, Goldberg MW \& Allen TD (1997). Three-dimensional visualization of the route of protein import: the role of nuclear pore complex substructures. Experimental Cell Research, 232: 146-160.

10. Oberleithner H, Brinckmann E, Schwab A \& Kröhne G (1994). Imaging nuclear pores of aldosterone-sensitive kidney cells by atomic force microscopy. Proceedings of the National Academy of Sciences, USA, 91: 9784-9788.

11. Greber UF \& Gerace L (1995). Depletion of calcium from the lumen of endoplasmic reticulum reversibly inhibits passive diffusion and signal-mediated transport into the nucleus. Journal of Cell Biology, 128: 5-14.

12. Perez-Terzic $C$, Jaconi $M$ \& Clapham DE (1997). Nuclear calcium and the regulation of the nuclear pore complex. Bioessays, 19: 787-792.

13. Bustamante JO, Hanover JA \& Liepins A (1995). The ion channel behavior of the nuclear pore complex. Journal of Membrane Biology, 146: 239-251.

14. Simon SM (1995). Protein-conducting channels for the translocation of proteins into and across membranes. Cold Spring Harbor Symposia on Quantitative Biology, 60: 57-69.

15. Paine PL \& Horowitz SB (1980). The movement of material between nucleus and cytoplasm. Cell Biology, 4: 299-338.

16. Miller M, Park MK \& Hanover JA (1991). Nuclear pore complex: structure, function, and regulation. Physiological Reviews, 71: 909-949.

17. Horowitz SB \& Paine PL (1976). Cytoplasmic exclusion as a basis for asymmetric nucleocytoplasmic solute distributions. Nature, 260: 151-153.

18. Paine PL (1993). Nuclear protein accumulation by facilitated transport and intranuclear binding. Trends in Cell Biology, 3 : 325-329.

19. Ling GN (1977). Potassium accumulation in frog muscle: the association-induction hypothesis versus the membrane theory. Science, 198: 1281-1284.

20. Ling GN (1994). The new cell physiology: an outline, presented against its full historical background, beginning from the beginning. Physiological Chemistry and Physics and Medical NMR, 26: 121-203.

21. Loewenstein WR \& Kanno Y (1962). Some electrical properties of the membrane of a cell nucleus. Nature, 195: 462-464.

22. Wiener J, Spiro D \& Loewenstein WR (1965). Ultrastructure and permeability of nuclear membranes. Journal of Cell Biology, 27: 107-117.

23. Matzke AJM, Weiger TM \& Matzke MA (1990). Detection of a large cation-selective channel in nuclear envelopes of avian erythrocytes. FEBS Letters, 271: 161-164.

24. Mazzanti M, DeFelice LJ, Cohen J \& Malter H (1990). lon channels in the nuclear envelope. Nature, 343: 764-767.

25. Bustamante JO (1994). Nuclear electrophysiology. Journal of Membrane Biology, 138: 105-112.

26. Bustamante JO, Liepins A \& Hanover JA (1994). Nuclear pore complex ion channels. Molecular Membrane Biology, 11: 141-150.

27. Dale B, DeFelice LJ, Kyozuka K, Santella L \& Tosti E (1994). Voltage clamp of the nuclear envelope. Proceedings of the Royal Society of London, Series B: Biological Sciences, 255: 119-124.

28. DeFelice LJ \& Mazzanti M (1995). Biophysics of the nuclear envelope. In: Sperelakis N (Editor), Cell Physiology. Academic Press, New York.

29. Stehno-Bittel L, Perez-Terzic C, Luckhoff A \& Clapham DE (1996). Nuclear ion channels and regulation of the nuclear pore. Society of General Physiologists Series, 51: 195-207.

30. Matzke AJM, Behensky C, Weiger T \&
Matzke MA (1992). A large conductance ion channel in the nuclear envelope of a higher plant cell. FEBS Letters, 302: 8185.

31. Bustamante JO (1992). Nuclear ion channels in cardiac myocytes. Pflügers $\mathrm{Ar}$ chives, European Journal of Physiology, 421: 473-485

32. Mazzanti M, DeFelice LJ \& Smith EF (1991). Ion channels in murine nuclei during early development and in fully differentiated adult cells. Journal of Membrane Biology, 121: 189-198.

33. Maruyama $Y$, Shimada $H$ \& Taniguchi $J$ (1995). $\mathrm{Ca}^{2+}$-activated $\mathrm{K}^{+}$-channels in the nuclear envelope isolated from single pancreatic acinar cells. Pflügers Archives, European Journal of Physiology, 430: 148150.

34. Guihard G, Proteau S \& Rousseau E (1997). Does the nuclear envelope contain two types of ligand-gated $\mathrm{Ca}^{2+}$ release channels? FEBS Letters, 414: 89-94.

35. Mak DO \& Foskett JK (1994). Single-channel inositol 1,4,5-trisphosphate receptor currents revealed by patch clamp of isolated Xenopus oocyte nuclei. Journal of Biological Chemistry, 269: 29375-29378.

36. Mak DO \& Foskett JK (1997). Single-channel kinetics, inactivation, and spatial distribution of inositol trisphosphate $\left(\mathrm{IP}_{3}\right)$ receptors in Xenopus oocyte nucleus. Journal of General Physiology, 109: 571-587.

37. Stehno-Bittel L, Luckhoff A \& Clapham DE (1995). Calcium release from the nucleus by $\mathrm{Ins}_{3}$ receptor channels. $\mathrm{Neu}$ ron, 14: 163-167.

38. Pasyk EA \& Foskett JK (1997). Cystic fibrosis transmembrane conductance regulator-associated ATP and adenosine 3'phosphate 5'-phosphosulfate channels in endoplasmic reticulum and plasma membranes. Journal of Biological Chemistry 272: 7746-7751.

39. Al-Mohanna FA, Caddy KW \& Bolsover SR (1994). The nucleus is insulated from large cytosolic calcium ion changes. $\mathrm{Na}$ ture, 367: 745-750.

40. Bustamante JO (1993). Restricted ion flow at the nuclear envelope of cardiac myocytes. Biophysical Journal, 64: 17351749.

41. Assandri R \& Mazzanti M (1997). Ionic permeability on isolated mouse liver nuclei: influence of ATP and $\mathrm{Ca}^{2+}$. Journal of Membrane Biology, 157: 301-309.

42. Hille $\mathrm{B}$ (1992). $\mathrm{Na}$ and $\mathrm{K}$ channels of axons. In: Hille B (Editor), Ionic Channels of Excitable Membranes. Chapter 3. 2nd edn. 
Sinauer, Sunderland, MA

43. Bustamante JO (1994). Open states of nuclear envelope ion channels in cardiac myocytes. Journal of Membrane Biology, 138: 77-89.

44. Moore MS \& Blobel G (1993). The GTPbinding protein Ran/TC4 is required for protein import into the nucleus. Nature, 365: 661-663.

45. Rout MP \& Blobel G (1993). Isolation of the yeast nuclear pore complex. Journal of Cell Biology, 123: 771-783.

46. Innocenti B \& Mazzanti M (1993). Identification of a nucleo-cytoplasmic ionic pathway by osmotic shock in isolated mouse liver nuclei. Journal of Membrane Biology, 131: 137-142.

47. Loewenstein WR, Kanno Y \& Ito S (1966). Permeability of the nuclear membrane. Annals of the New York Academy of Sciences, 137: 708-716.

48. Tabares L, Mazzanti M \& Clapham DE (1991). Chloride channels in the nuclear membrane. Journal of Membrane Biology, 123: 49-54.

49. Longin AS, Mezin P, Favier A \& Verdetti J (1997). Presence of zinc and calcium permeant channels in the inner membrane of the nuclear envelope. Biochemical and Biophysical Research Communications, 235: 236-241.

50. Draguhn A, Börner G, Beckmann R, Buchner $K$, Heinemann $U$ \& Hucho $F$ (1997). Large-conductance cation channels in the envelope of nuclei from rat cerebral cortex. Journal of Membrane Biology, 158: 159-166.

51. Rousseau E, Michaud C, Lefebvre D, Proteau S \& Decrouy A (1996). Reconstitution of ionic channels from inner and outer membranes of mammalian cardiac nuclei. Biophysical Journal, 70: 703-714.

52. Valenzuela SM, Martin DK, Por SB, Robbins JM, Warton K, Bootcov MR, Schofield PR, Campbell TJ \& Breit SN (1997). Molecular cloning and expression of a chloride ion channel of cell nuclei. Journal of Biological Chemistry, 272: 12575-12582.

53. Stehno-Bittel L, Perez-Terzic C \& Clapham DE (1995). Diffusion across the nuclear envelope inhibited by depletion of nuclear $\mathrm{Ca}^{2+}$ store. Science, 270: 1835-1838.

54. Hinshaw JE, Carragher BO \& Milligan RA (1992). Architecture and design of the nuclear pore complex. Cell, 69: 11331141.

55. Tomalia DA (1995). Dendrimer molecules. Scientific American, 272: 62-65.

56. Perez-Terzic C, Stehno-Bittel L \& Clapham DE (1997). Nucleoplasmic and cytoplas- mic differences in the fluorescence properties of the calcium indicator Fluo-3. Cell Calcium, 21: 275-282.

57. Prat AG \& Cantiello H (1996). Nuclear ion channel activity is regulated by actin filaments. American Journal of Physiology, 270: C1532-C1543.

58. Lohret $T A$, Jensen RE \& Kinnally KW (1997). Tim23, a protein import component of the mitochondrial inner membrane, is required for normal activity of the multiple conductance channel, MCC. Journal of Cell Biology, 137: 377-386.

59. Coronado R, Morrissette J, Sukhareva M \& Vaughan DM (1994). Structure and function of ryanodine receptors. American Journal of Physiology, 266: C1485-C1504.

60. Lohret TA \& Kinnally KW (1995). Targeting peptides transiently block a mitochondrial channel. Journal of Biological Chemistry, 270: 15950-15953.

61. Bustamante $\mathrm{JO}$, Oberleithner $\mathrm{H}$, Hanover JA \& Liepins A (1995). Patch clamp detection of transcription factor translocation along the nuclear pore complex channel. Journal of Membrane Biology, 146: 253261.

62. Bustamante JO, Liepins A, Prendergast RA, Hanover JA \& Oberleithner H (1995). Patch clamp and atomic force microscopy demonstrate TATA-binding protein (TBP) interactions with the nuclear pore complex. Journal of Membrane Biology, 146: 263-272.

63. Jans DA \& Hubner S (1996). Regulation of protein transport to the nucleus: central role of phosphorylation. Physiological Reviews, 76: 651-685.

64. Mazzanti $M$, Innocenti B \& Rigatelli $M$ (1994). ATP-dependent ionic permeability on nuclear envelope in in situ nuclei of Xenopus oocytes. FASEB Journal, 8: 231236.

65. Henry JP, Juin P, Vallette F \& Thieffry M (1996). Characterization and function of the mitochondrial outer membrane peptide-sensitive channel. Journal of Bioenergetics and Biomembranes, 28: 101-108.

66. Pelleschi M, Henry JP \& Thieffry M (1997). Inactivation of the peptide-sensitive channel from the yeast mitochondrial outer membrane: properties, sensitivity to trypsin and modulation by a basic peptide. Journal of Membrane Biology, 156: 37-44.

67. Jurgens L, Kleineke J, Brdiczka D, Thinnes FP \& Hilschmann N (1995). Localization of type-1 porin channel (VDAC) in the sarcoplasmatic reticulum. Biological Chemistry Hoppe-Seyler, 376: 685-689.

68. Colombini M, Blachly-Dyson E \& Forte M
(1996). VDAC, a channel in the outer mitochondrial membrane. Ion Channels, 4: 169-202.

69. Jensen RE \& Kinnally KW (1997). The mitochondrial protein import pathway: are precursors imported through membrane channels? Journal of Bioenergetics and Biomembranes, 29: 3-10.

70. Crowley KS, Liao S, Worrell VE, Reinhart GD \& Johnson AE (1994). Secretory proteins move through the endoplasmic reticulum membrane via an aqueous, gated pore. Cell, 78: 461-471.

71. Hamman BD, Chen J-C, Johnson EE \& Johnson AE (1997). The aqueous pore through the translocon has a diameter of 40-60 A during cotranslational protein translocation at the ER membrane. Cell, 89: 535-544.

72. Perez-Terzic C, Pyle J, Jaconi M, StehnoBittel L \& Clapham DE (1996). Conformational states of the nuclear pore complex induced by depletion of nuclear $\mathrm{Ca}^{2+}$ stores. Science, 273: 1875-1877.

73. Badminton MN, Kendall JM, Sala-Newby G \& Campbell AK (1995). Nucleoplasmintargeted aequorin provides evidence for a nuclear calcium barrier. Experimental Cell Research, 216: 236-243.

74. Badminton MN, Campbell AK \& Rembold CM (1996). Differential regulation of nuclear and cytosolic $\mathrm{Ca}^{2+}$ in HeLa cells. Journal of Biological Chemistry, 271: 31210-31214.

75. Dupont G, Pontes J \& Goldbeter A (1996). Modeling spiral $\mathrm{Ca}^{2+}$ waves in single cardiac cells: role of the spatial heterogeneity created by the nucleus. American Journal of Physiology, 271: C1390-C1399.

76. Gerasimenko OV, Gerasimenko JV, Petersen OH \& Tepikin AV (1996). Short pulses of acetylcholine stimulation induce cytosolic $\mathrm{Ca}^{2+}$ signals that are excluded from the nuclear region in pancreatic acinar cells. Pflügers Archives, European Journal of Physiology, 432: 1055-1061.

77. Kong SK, Tsang D, Leung KN \& Lee CY (1996). Nuclear envelope acts as a calcium barrier in $\mathrm{C} 6$ glioma cells. Biochemical and Biophysical Research Communications, 218: 595-600.

78. Seksek O \& Bolard J (1996). Nuclear pH gradient in mammalian cells revealed by laser microspectrofluorimetry. Journal of Cell Science, 109: 257-262.

79. Santella L (1996). The cell nucleus: an Eldorado to future calcium research? Journal of Membrane Biology, 153: 83-92.

80. Bachs O, Carafoli E, Nicotera R \& Santella L (Organizers) (1997). First European Conference on Calcium Signalling in the Cell 
Nucleus. European Commission DG XII, Biotechnology Programme, October 4-8, Baia Paraelios, Calabria, Italy 4-8 October, 1997.

81. Brini M, Marsault R, Bastianutto C, Pozzan T \& Rizzuto R (1994). Nuclear targeting of aequorin. A new approach for measuring nuclear $\mathrm{Ca}^{2+}$ concentration in intact cells. Cell Calcium, 16: 259-268.

82. Miyashita $Y$, Miller M, Yen PM, Harmon JM, Hanover JA \& Simons Jr SS (1993). Glucocorticoid receptor binding to rat liver nuclei occurs without nuclear transport. Journal of Steroid Biochemistry and Molecular Biology, 46: 309-320.

83. Haltiwanger RS, Busby S, Grove K, Li S, Mason D, Medina L, Moloney D,
Philipsberg G \& Scartozzi R (1997). O glycosylation of nuclear and cytoplasmic proteins: regulation analogous to phosphorylation? Biochemical and Biophysical Research Communications, 231:237-242.

84. Bezrukov SM, Vodyanoy I \& Parsegian VA (1994). Counting polymers moving through a single ion channel. Nature, 370: 279-281.

85. Daneholt B (1997). A look at messenger RNP moving through the nuclear pore. Cell, 88: 585-588.

86. Citovsky V \& Zambryski P (1993). Transport of nucleic acids through membrane channels: snaking through small holes. Annual Review of Microbiology, 47: 167197.
87. Dean DA (1997). Import of plasmid DNA into the nucleus is sequence specific. $E x$ perimental Cell Research, 230: 293-302.

88. Kasianowicz JJ, Brandin E, Branton D \& Deamer DW (1996). Characterization of individual polynucleotide molecules using a membrane channel. Proceedings of the National Academy of Sciences, USA, 93: 13770-13773.

89. Krasilnikov OV, Carneiro CM, Yuldasheva LN, Campos de Carvalho AC \& Nogueira RA (1996). Diameter of the mammalian porin channel in open and "closed" states: direct measurement at the single channel level in planar lipid bilayer. Brazilian Journal of Medical and Biological Research, 29: 1691-1697. 